\title{
Atypical Regeneration
}

National Cancer Institute

\section{Source}

National Cancer Institute. Atypical Regeneration. NCI Thesaurus. Code C17945.

Atypical Regeneration consists of reactive cellular proliferation, abnormal reproduction, or renewal of cells and tissues that have been damaged or destroyed due to injury, morbidity, or ordinary life processes and is characterized by nuclear/cytoplasmic atypia. The concept encompasses size, shape, and org anization of cell architecture specific for dysplasia, cancer in situ, inflammatory atypia, and preneoplastic states associated with regenerative changes in tissue. 